\title{
Time-to-space mapping in a gas medium for the temporal characterization of vacuum-ultraviolet pulses
}

\author{
S. Cunovic and N. Müller \\ Department of Physics, Bielefeld University, Universitätsstrasse 25, D-33615 Bielefeld, Germany \\ R. Kalms, M. Krikunova, M. Wieland, and M. Drescher ${ }^{\mathrm{a})}$ \\ Institut für Experimentalphysik, University of Hamburg, Luruper Chaussee 149, D-22761 Hamburg, \\ Germany
}

Th. Maltezopoulos, U. Frühling, H. Redlin, E. Plönjes-Palm, and J. Feldhaus

HASYLAB at DESY, Notkestrasse 85, D-22607 Hamburg, Germany

(Received 4 December 2006; accepted 13 February 2007; published online 21 March 2007)

\begin{abstract}
The authors introduce a method for cross correlating vacuum-ultraviolet with near-infrared femtosecond light pulses in a perpendicular geometry. Photoelectrons generated in an atomic gas by laser-assisted photoionization are used to create a two-dimensional image of the cross-correlation volume, thereby mapping time onto a space coordinate. Thus, information about pulse duration and relative timing between the pulses can be obtained without the need to scan an optical delay line. First tests using vacuum-ultraviolet pulses from the free-electron laser at the Deutsches Elektronen Synchrotron set an upper limit for their temporal jitter with respect to external optical laser pulses. (C) 2007 American Institute of Physics. [DOI: 10.1063/1.2714999]
\end{abstract}

For measurement of the temporal properties of optical pulses in the visible, near-ultraviolet, and near-infrared (NIR) range the nonlinear response of an optical medium-usually a crystal - on two optical fields of different or the same color is often used to realize cross- or autocorrelation schemes. ${ }^{1}$ The recent advent of sources of femtosecond-and even attosecond $^{2}$ - pulses of ionizing radiation, based on laser plasmas, ${ }^{3}$ high harmonic generation, ${ }^{4}$ and free-electron lasing calls for a transfer of the correlation principle to the vacuum-ultraviolet (vuv) and the x-ray range. Since, however, crystals are no longer transparent in the vuv and do not exhibit sufficient nonlinearity in the x-ray range, alternative approaches for pulse characterization are necessary. Freeelectron lasers (FELs) are the most powerful femtosecond laser sources at short wavelengths, currently operating in the vuv range [e.g., free-electron laser Hamburg (FLASH) $]^{5}$ and down to X-rays in the future. ${ }^{6,7}$ So far, the utilized mode of operation for short-wavelength FELs relies on the process of self-amplified spontaneous emission (SASE). ${ }^{8}$ High photon energies combined with greatly enhanced pulse intensities as compared to other sources make FELs a promising source for time-resolved visible/vuv pump-probe studies. FELs, however, are based on linear accelerators and undulators, which together measure from a few hundred meters up to a few kilometers in length. This makes these instruments susceptible to path length variations on a micrometer scale. Moreover, the statistical nature of SASE comes with a frequently varying spectral and temporal profile for each individual shot. ${ }^{9}$ As a result, the arrival time of the FEL pulses at the experiment fluctuates in the order of a few hundred femtoseconds. This jitter inhibits an exact synchronization to an external laser source needed for reliable pump-probe studies. Therefore, an adequate characterization method is needed to determine the pulse duration together with the actual delay with respect to an external laser on a shot-to-shot basis. State-of-the-art x-ray streak cameras have reached subpicosecond temporal resolution, ${ }^{10}$ but the photocathode com-

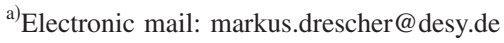

pletely dissipates the beam. In addition, their readout rate is limited to a few hertz, while FLASH is operated at a pulse rate up to $1 \mathrm{MHz}$. The recently introduced technique of electro-optical sampling is noninvasive and can be operated at high repetition rates. ${ }^{11}$ This measurement, however, takes place at a large distance from the experiment and correlates NIR laser with electron rather than vuv pulses. We present a cross-correlation scheme for the direct temporal characterization of vuv or $\mathrm{x}$-ray pulses using a specific geometry that maps temporal information onto a spatial coordinate, thereby exploiting the potential for a single-shot measurement. In contrast to a different approach, where x-ray diffraction from a crystal was utilized, ${ }^{12}$ the principle relies on the simultaneous interaction of the vuv and a visible laser pulse in a gas medium, making the method noninvasive for both pulses.

The results were obtained at FLASH operating at a wavelength of $\lambda=32 \mathrm{~nm}(h \nu \approx 39 \mathrm{eV})$ with micropulse energies of $E_{p}=2-15 \mu \mathrm{J}$. An optical laser system with $800 \mathrm{~nm}$ wavelength $(h \nu \approx 1.5 \mathrm{eV})$, a pulse duration of $\approx 150 \mathrm{fs}$, and a pulse energy of up to $15 \mu \mathrm{J}$ is electronically synchronized with FLASH based on the $1.3 \mathrm{GHz}$ master clock of the facility. Both lasers share the same temporal pulse pattern consisting of $n_{\text {micro }}=1-30$ micropulses separated by $1 \mu \mathrm{s}$ from each other forming a macropulse (burst) with a repetition rate of $5 \mathrm{~Hz}$.

The experimental setup is depicted schematically in Fig. 1. The unfocused vuv pulse of $\approx 7 \mathrm{~mm}$ [full width at half maximum (FWHM)] in diameter enters the target chamber through an entrance slit of $500 \mu \mathrm{m}$ height and $150 \mu \mathrm{m}$ width. A krypton gas target is supplied by a gas nozzle (chamber pressure of $1 \times 10^{-5} \mathrm{mbar}$, without gas of $\left.1 \times 10^{-8} \mathrm{mbar}\right)$. The FEL beam produces photoelectrons with a kinetic energy $E_{\text {kin }}=h \nu_{\text {FEL }}-W_{b}$ along its path, $W_{b}$ denoting the ionization potential of the target gas. The NIR laser pulses propagate perpendicularly to the FEL and are focused to $\approx 20 \mu \mathrm{m}(\mathrm{FWHM})$ in front of the entrance aperture of the electron optical system. Both beams are linearly polarized parallel to the axis of the electron optics. In the interaction region both pulses overlap in space and time. Its geometrical 


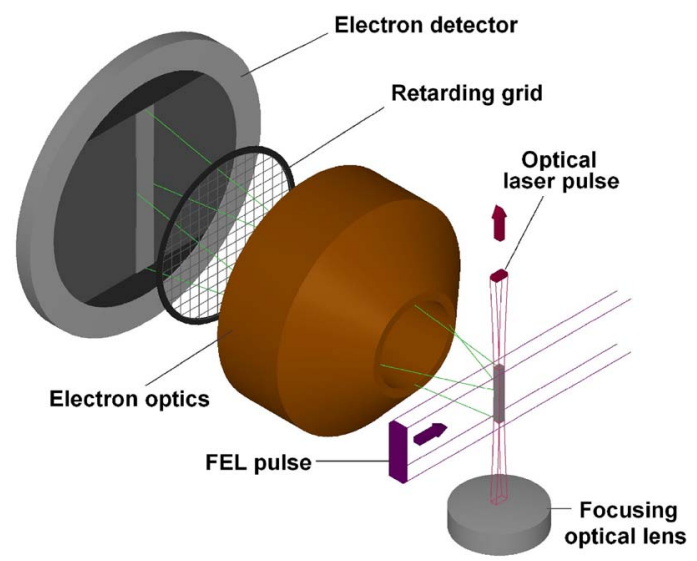

FIG. 1. (Color online) Experimental setup: Pulses from FLASH and a NIR laser are superimposed in a $\mathrm{Kr}$ gas target. The region of temporal and spatial overlap is imaged by an electron lens system with a high-pass energy filter.

height of $500 \mu \mathrm{m}$ corresponds to a time window of $1.7 \mathrm{ps}$. Photoelectrons originating from this region can be energetically shifted due to the interaction with the NIR laser field (laser assisted photoionization). ${ }^{13}$ The energy exchange occurs in a quantized manner, leading to spectral sidebands in the photoelectron energy spectrum separated from the unperturbed photoline by multiple integers of the NIR laser's photon energy $\Delta E= \pm n h \nu{ }^{14}$

The area around the interaction region is imaged by means of an energy-filtering electron lens system at a magnification of $M \approx 20$, corresponding to a field of view of about $700 \mu \mathrm{m}$. The electrons are detected with a double microchannel plate and transformed into an optical image with a phosphor screen monitored by a charge coupled device camera. A grid in the electron path realizes a high-pass energy filter, separating energetically upshifted electrons from unperturbed photoelectrons by applying a retarding voltage $U_{\text {ret. }}$ The area-integrated photoelectron signal without NIR laser field is shown in Fig. 2 as a function of $U_{\text {ret. }}$ The appropriate value of $U_{\text {ret }}$ for separating the positive sidebands $(\Delta E>0)$ from the main photoline is referred to as cutoff voltage $U_{\text {cut }}$. It can be deduced from the derivative of the signal showing a single photoline with a width of $\approx 1 \mathrm{eV}$. The inset in Fig. 2 shows the derivative of the signal with optical laser on. As expected, spectral sidebands appear with

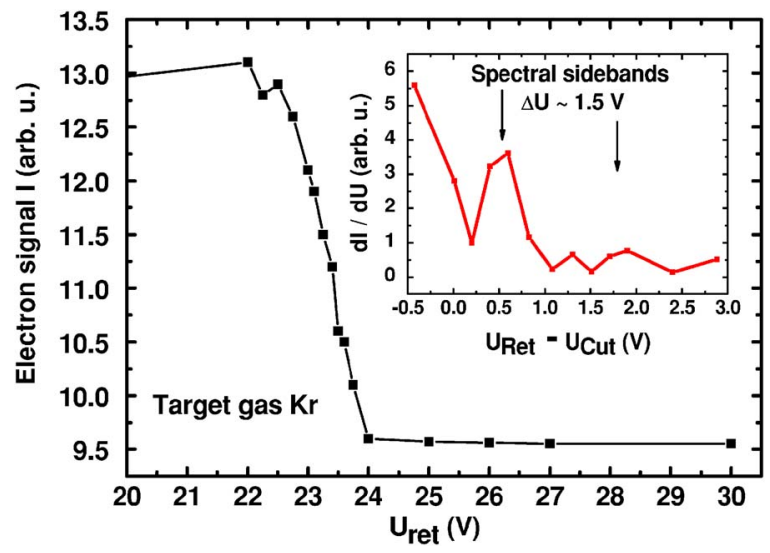

FIG. 2. (Color online) Area-integrated photoelectron signal from $\mathrm{Kr}$ atoms ionized with $39 \mathrm{eV}$ vuv pulses plotted against retarding voltage in the absence of an optical laser field. Inset: Derivative of the photoelectron signal above $U_{\text {cut }}$ with the optical laser on. Every data point is averaged over 200

sures, each illuminated by one macropulse $\left(n_{\text {micro }}=30\right)$.
macropulses $\left(n_{\text {micro }}=30\right)$.
Downloaded 27 Jun 2007 to 131.169.95.147. Redistribution subject to AlP license or copyright, see http://apl.aip.org/a

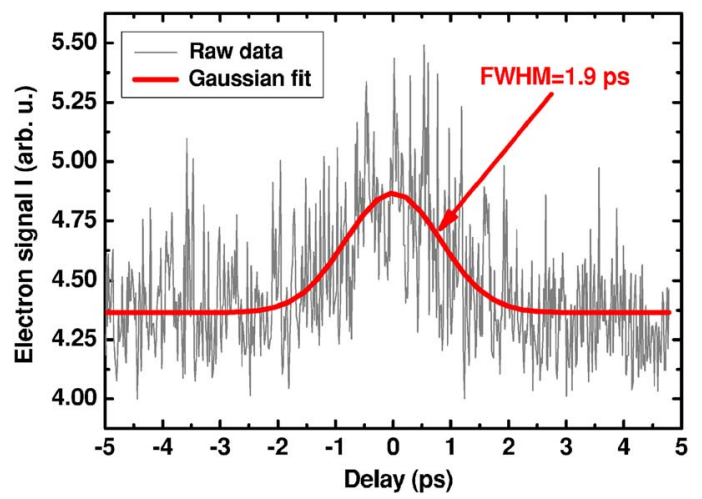

FIG. 3. (Color online) Area-integrated photoelectron signal at $U_{\text {cut }}$ plotted against relative vuv/NIR timing. Signal from spectral sidebands appears only for a temporal overlap of both pulses. Every data point corresponds to one macropulse $\left(n_{\text {micro }}=30\right)$.

a $1.5 \mathrm{eV}$ energy difference. The precondition to acquire this signal is a precise spatial and temporal overlap of both pulses in the interaction region. The spatial overlap is monitored with an accuracy of $\sim 25 \mu \mathrm{m}$ using a fluorescence screen for the vuv and several optical imaging systems for the NIR radiation. The temporal prealignment is accomplished with $\sim 10$ ps accuracy using a high-bandwidth copper photocathode, which can be placed into the interaction region. With the two beams properly prealigned in space and time, a NIR delay line scan over \pm 10 ps (Fig. 3) revealed additional electron signal at a distinct delay setting, now denoted as time zero. The kinetic energy spectrum of this feature presented in the inset in Fig. 2 exhibits the peaked structure at energetic positions expected for NIR-induced sidebands with $n=1,2$.

Typical electron optical images obtained at $\Delta t=0 \mathrm{ps}$ after summation over several pulses are presented in Fig. 4. Figure 4(a) shows the channel created by ionization of krypton atoms with vuv radiation from FLASH without a NIR laser field at $U_{\text {ret }}<U_{\text {cut }}$. The image of the channel almost vanishes for $U_{\text {ret }}=U_{\text {cut }}$ [Fig. 4(b)]; the remaining signal is a good indicator for the noise background of this measurement. When the NIR laser is superimposed, signal reappears in the interaction region. This is even more clearly
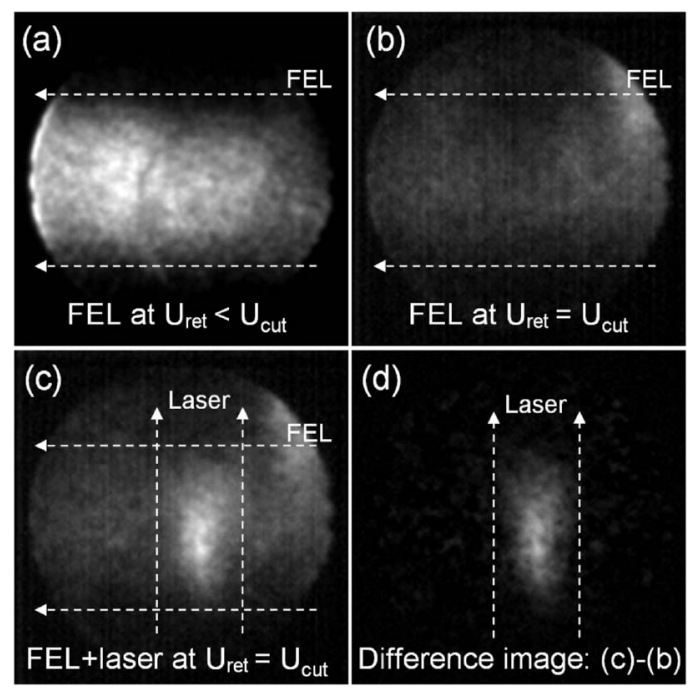

FIG. 4. Photoelectron images: (a) $U_{\text {ret }}<U_{\text {cut }}$ (FEL ionization path without optical laser), (b) residual signal at $U_{\text {ret }}=U_{\text {cut }}$ without optical laser, (c) same as (b) but with optical laser on, and (d) difference picture (c)-(b). Images are generated by summation of (a) 200 or [(b)-(d)] 1200 individual expo-

sures, each illuminated by one macropulse $\left(n_{\text {micro }}=30\right)$.
o AIP license or copyright, see http://apl.aip.org/apl/copyright.jsp 
seen in the difference image in Fig. 4(d) obtained by subtracting Fig. 4(b) from Fig. 4(c).

Only such electrons are imaged that have been set free from the gas atoms when simultaneously the NIR field was present. The obtained two-dimensional image reflects the form and relative spatial position of the vuv and NIR wave packets. Since the NIR temporal pulse profile can be measured independently using standard cross-correlation techniques, the vuv pulse duration and its relative jitter with respect to the NIR laser pulses can be deduced. It should be noted, however, that in the currently realized setup, the formation of a meaningful image requires a summation over many individual pulses. Correspondingly, the captured images represent an average over many different delays and thereby provide an upper limit for the vuv/NIR jitter. Fitting of the peak in Fig. 3 with a Gaussian envelope results in a temporal width of 1.9 ps. This value results from the convolution of the experimental time window, the jitter, and the pulse widths of the vuv and NIR pulses. Since the latter contribute with only $\approx 30$ fs (Ref. 5) and $\approx 150$ fs, respectively, the measured width can be regarded as an upper limit for the jitter. Recently, the remaining rms jitter for the current machine setup has been determined with a different crosscorrelation approach for pump-probe experiments to be less than 1 ps. ${ }^{15}$

In conclusion, we present a technique for cross correlating light pulses of very different wavelengths in the NIR $(\lambda=800 \mathrm{~nm})$ and the vuv $(\lambda=32 \mathrm{~nm})$ range. The use of a dilute gaseous medium makes the setup transparent for both pulses and virtually indestructible at any intensity. An orthogonal geometry allows for mapping time into space, thus eliminating the need for scanning a temporal delay in subsequent pulses. While this feature in principle facilitates a single-shot analysis of both pulse duration and vuv/NIR delay, the present signal level requires averaging over many pulses in order to obtain sufficient signal. A Monte-Carlo simulation using the SIMION $^{16}$ software for propagating a statistically distributed set of electrons through the imaging system shows that 100 sideband electrons at the detector will provide sufficient signal-to-noise ratio for determining the delay with $20 \mathrm{fs}$ and the pulse duration with $80 \mathrm{fs}$ precision. This requires a 50-fold enhancement over the signal level utilized in this work. Consequently, an extension to singleshot analysis is within reach by increasing either the target density (factor of ten is possible) or the vuv pulse energy in the interaction region (focusing will yield a factor of 100), provided that space charge effects can be kept at a tolerable level. The recently demonstrated operation of FLASH at $13 \mathrm{~nm}$ wavelength will result in higher kinetic energies of the emitted photoelectrons, resulting in an even more efficient sideband formation. ${ }^{2}$ In fact, the underlying physical mechanism-photoionization of a free atom-works at any photon energy exceeding the ionization potential, making the method extendable also to extremely short wavelengths, as will be delivered by future FEL facilities like the Linac Coherent Light Source and the X-FEL.

The authors thank the FEL machine group and the photon diagnostics team, especially S. Düsterer and A. Azima. Financial support from the EU Project No. IA-SFS JRA2 and the HGF virtual institute "accelerator metrology" is gratefully acknowledged.
${ }^{1}$ G. Steinmeyer, J. Opt. A, Pure Appl. Opt. 5, R1 (2003).

${ }^{2}$ M. Drescher, M. Hentschel, R. Kienberger, G. Tempea, Ch. Spielmann, G. A. Reider, P. B. Corkum, and F. Krausz, Science 291, 1923 (2001).

${ }^{3}$ D. von der Linde and K. Sokolowski-Tinten, J. Mod. Opt. 50, 683 (2003).

${ }^{4}$ T. Brabec and F. Krausz, Rev. Mod. Phys. 72, 545 (2000).

${ }^{5}$ V. Ayvazyan, N. Baboi, J. Bähr, V. Balandin, B. Beutner, A. Brandt, I. Bohnet, A. Bolzmann, R. Brinkmann, O. I. Brovko, J. P. Carneiro, S. Casalbuoni, M. Castellano, P. Castro, L. Catani, E. Chiadroni, S. Choroba, A. Cianchi, H. Delsim-Hashemi, G. Di Pirro, M. Dohlus, S. Düsterer, H. T. Edwards, B. Faatz, A. A. Fateev, J. Feldhaus, K. Flöttmann, J. Frisch, L. Fröhlich, T. Garvey, U. Gensch, N. Golubeva, H.-J. Grabosch, B. Grigoryan, O. Grimm, U. Hahn, J. H. Han, M. v. Hartrott, K. Honkavaara, M. Hüning, R. Ischebeck, E. Jaeschke, M. Jablonka, R. Kammering, V. Katalev, B. Keitel, S. Khodyachykh, Y. Kim, V. Kocharyan, M. Körfer, M. Kollewe, D. Kostin, D. Krämer, M. Krassilnikov, G. Kube, L. Lilje, T. Limberg, D. Lipka, F. Löhl, M. Luong, C. Magne, J. Menzel, P. Michelato, V. Miltchev, M. Minty, W. D. Möller, L. Monaco, W. Müller, M. Nagl, O. Napoly, P. Nicolosi, D. Nölle, T. Nuñez, A. Oppelt, C. Pagani, R. Paparella, B. Petersen, B. Petrosyan, J. Pflüger, P. Piot, E. Plönjes, L. Poletto, D. Proch, D. Pugachov, K. Rehlich, D. Richter, S. Riemann, M. Ross, J. Rossbach, M. Sachwitz, E. L. Saldin, W. Sandner, H. Schlarb, B. Schmidt, M. Schmitz, P. Schmüser, J. R. Schneider, E. A. Schneidmiller, H.-J. Schreiber, S. Schreiber, A. V. Shabunov, D. Sertore, S. Setzer, S. Simrock, E. Sombrowski, L. Staykov, B. Steffen, F. Stephan, F. Stulle, K. P. Sytchev, H. Thom, K. Tiedtke, M. Tischer, R. Treusch, D. Trines, I. Tsakov, A. Vardanyan, R. Wanzenberg, T. Weiland, H. Weise, M. Wendt, I. Will, A. Winter, K. Wittenburg, M. V. Yurkov, I. Zagorodnov, P. Zambolin, and K. Zapfe, Eur. Phys. J. D 37, 297 (2006).

${ }^{6}$ P. Audebert, V. Balandin, W. Bialowons, A. R. B. de Castro, S. Choroba, H.-K. Chung, R. W. Falcone, J. Feldhaus, H. Franz, T. E. Glover, W. Graeff, N. Golubeva, P. Gürtler, U. Hahn, J. Hajdu, L. Hänisch, R.-D. Heuer, G. Huldt, J.-P. Jensen, S. Johnson, T. Laarmann, W. Laasch, R. W. Lee, A. Leuschner, A. M. Lindenberg, Th. Möller, W. L. Morgan, F. Peters, J. Pflüger, D. Proch, D. Riley, W. Rozmus, E. Saldin, M. Schmitz, E. Schneidmiller, J. Schulz, A. Schwarz, D. van der Spoel, F. Stephan, J. Sutter, A. Szöke, S. Techert, N. Tesch, M. Tischer, H. Wabnitz, E. Weckert, M. Yurkov, and B. Ziaja, Report No. DESY 2002-167, 2002 (unpublished); http://xfel.desy.de

${ }^{7}$ C. Limborg, Nucl. Instrum. Methods Phys. Res. A 507, 378 (2003).

${ }^{8}$ A. M. Kondratenko and E. L. Saldin, Part. Accel. 10, 207 (1980).

${ }^{9}$ J. Rossbach, Nucl. Instrum. Methods Phys. Res. A 507, 362 (2003).

${ }^{10}$ R. Shepherd, R. Booth, D. Price, M. Bowers, D. Swan, J. Bonlie, B. Young, J. Dunn, B. White, and R. Stewart, Rev. Sci. Instrum. 66, 719 (1995).

${ }^{11}$ A. L. Cavalieri, D. M. Fritz, S. H. Lee, P. H. Bucksbaum, D. A. Reis, J. Rudati, D. M. Mills, P. H. Fuoss, G. B. Stephenson, C. C. Kao, D. P. Siddons, D. P. Lowney, A. G. MacPhee, D. Weinstein, R. W. Falcone, R. Pahl, J. Als-Nielsen, C. Blome, S. Düsterer, R. Ischebeck, H. Schlarb, H. Schulte-Schrepping, Th. Tschentscher, J. Schneider, O. Hignette, F. Sette, K. Sokolowski-Tinten, H. N. Chapman, R. W. Lee, T. N. Hansen, O. Synnergren, J. Larsson, S. Techert, J. Sheppard, J. S. Wark, M. Bergh, C. Caleman, G. Huldt, D. van der Spoel, N. Timneanu, J. Hajdu, R. A. Akre, E. Bong, P. Emma, P. Krejcik, J. Arthur, S. Brennan, K. J. Gaffney, A. M. Lindenberg, K. Luening, and J. B. Hastings, Phys. Rev. Lett. 94, 114801 (2005).

${ }^{12}$ A. M. Lindenberg, J. Larsson, K. Sokolowski-Tinten, K. J. Gaffney, C. Blome, O. Synnergren, J. Sheppard, C. Caleman, A. G. MacPhee, D. Weinstein, D. P. Lowney, T. K. Allison, T. Matthews, R. W. Falcone, A. L. Cavalieri, D. M. Fritz, S. H. Lee, P. H. Bucksbaum, D. A. Reis, J. Rudati, P. H. Fuoss, C. C. Kao, D. P. Siddons, R. Pahl, J. Als-Nielsen, S. Düsterer, R. Ischebeck, H. Schlarb, H. Schulte-Schrepping, Th. Tschentscher, J. Schneider, D. von der Linde, O. Hignette, F. Sette, H. N. Chapman, R. W. Lee, T. N. Hansen, S. Techert, J. S. Wark, M. Bergh, G. Huldt, D. van der Spoel, N. Timneanu, J. Hajdu, R. A. Akre, E. Bong, P. Krejcik, J. Arthur, S. Brennan, K. Luening, and J. B. Hastings, Science 308, 392 (2005).

${ }^{13}$ T. E. Glover, R. W. Schoenlein, A. H. Chin, and C. V. Shank, Phys. Rev. Lett. 76, 2468 (1996).

${ }^{14} \mathrm{P}$. O'Keeffe, R. López-Martens, J. Mauritsson, A. Johansson, A. L'Huillier, V. Véniard, R. Taïeb, A. Maquet, and M. Meyer, Phys. Rev. A 69, 051401 (2004).

${ }^{15}$ P. Radcliffe, S. Düsterer, A. Azima, H. Redlin, J. Feldhaus, J. T. Costello, E. T. Kennedy, D. Cubaynes, and M. Meyer, Appl. Phys. Lett. (submitted).

${ }^{16} \mathrm{http}: / /$ www.simion.com/ 